\title{
New periods of variable stars in Cygnus based on a quarter of century of photographic observations ${ }^{\star}$
}

\author{
L. Hric ${ }^{1}$ and R. Gális ${ }^{1}$ \\ Astronomical Institute of the Slovak Academy of Sciences, 05960 Tatranská Lomnica, The Slovak Republic \\ e-mail: hric@ta3.sk galis@ta3.sk
}

Received March 13; accepted September 22, 1998

\begin{abstract}
This work summarizes the results of photographic photometry obtained over 24 years at the Skalnaté Pleso (1979-1993) and Asiago (1969-1988) observatories. The period of light changes, the epoch of the maximum or of the minimum, the type of variability and, in some cases, possible causes of the long-term behaviour of light changes were determined for 17 variable stars.
\end{abstract}

Key words: stars: techniques: photometric — stars: variables: general

\section{The observational material}

The symbiotic star V1329 Cyg had been observed during the years 1979 - 1993 using the $30 / 150 \mathrm{~cm}$ astrograph of the Skalnaté Pleso Observatory. Ninety photographic plates of $9 \times 12 \mathrm{~cm}$ and $24 \times 24 \mathrm{~cm}$ formats with emulsions ORWO ZU-2 and ORWO ZU-21 were obtained. The limiting magnitude of the observational material is about $16.5 \mathrm{~m}_{\mathrm{pg}}$. The resulting magnitude scale is close to the photoelectric $B$ magnitude. The results obtained for this symbiotic star were published by Hric et al. (1993). Because the region thereby investigated is situated only $6^{\circ}$ under the plane of the Milky Way, an idea has originated to make use of the huge amount of the exposed stars on photographic plates for the search of the accessible variable stars with the aim of the determination of their parameters.

Similar research had been underway already in the years 1969 - 1988 at the Asiago Observatory, where 85 photographic plates were obtained using the Schmidt telescope $(67 / 92 / 215 \mathrm{~cm})$ of the $24 \times 24 \mathrm{~cm}$ format with the

Send offprint requests to: L. Hric

* Tables 2 is only and Table 3 also available at the CDS via anonymous ftp 130.79.128.5 and http://cdsweb.ustrasbg.fr/Abstract.html emulsion Kodak 103a-O. The resulting photographic magnitude was close to the photoelectric $B$ magnitude. On the basis of this material, Margoni and Stagni (1984), hereafter MS, and Margoni et al. (1989), hereafter MSMM, found 99 new variable stars in the field under investigation and determined their photographic magnitudes, their light curves (LCs) and, for many of them, also their periods. Thanks to Margoni (1993), who has provided us with his original material in the reduced form, we were able to extend our observational material and almost to double the time interval investigated by us.

During the complex survey of the combined observational material, we have identified and selected 17 variable stars present on almost all the photographic plates and fitting the conditions required for obtaining of photographic magnitudes (the plate's edge, limiting stellar magnitude). We used the findig charts published in MS. Only 3 of those stars were described in the GCVS (Kholopov et al. 1985), further 11 stars were described in The 68th Name-List of Variable Stars (Kholopov et al. 1987). For stars selected that way, we have determined photographic magnitudes through the remeasurement using the iris microphotometer at the Asiago Observatory. Nine stars from the close neighbourhood of V1329 Cygni whose magnitudes were measured by Kohoutek (1969) were used as comparisons, as well as those used as comparisons by MS, 18 objects in total. The list of all comparison stars with $B$ magnitude and source is presented in Table 1 . A large number of comparisons should be made to get a better definition of the sensitometric characteristic for each plate as well as to suppress errors of photographic photometry. We used the same finding chart like MS for comparisons and finding chart as well as $U B V$ magnitudes from Grygar et al. (1979) for nine comparisons by Kohoutek (1969). In addition to the stars measured, also the sky level was measured (meaning the combination photographic plate foglevel and the real sky brightness) in the vicinity of each star measured. Almost 3000 measurements (variables, comparisons) were performed. 
Table 1. The list of comparison stars

\begin{tabular}{llll}
\hline \hline Stars by Kohoutek (1969) & $m_{B}$ & Stars by MS & $m_{B}$ \\
\hline $\mathrm{a}=\mathrm{BD}+354294$ & 11.23 & $\mathrm{a}$ & 10.20 \\
$\mathrm{~b}=\mathrm{BD}+354290$ & 11.41 & $\mathrm{~b}$ & 11.70 \\
$\mathrm{c}$ & 12.28 & $\mathrm{c}$ & 12.05 \\
$\mathrm{~d}$ & 13.00 & $\mathrm{~d}$ & 12.85 \\
$\mathrm{e}$ & 13.39 & $\mathrm{e}$ & 13.90 \\
$\mathrm{f}$ & 14.41 & $\mathrm{f}$ & 14.95 \\
$\mathrm{~g}$ & 14.48 & $\mathrm{~g}$ & 15.80 \\
$\mathrm{~h}$ & 15.90 & $\mathrm{~h}$ & 16.60 \\
$\mathrm{i}$ & 16.20 & $\mathrm{l}$ & 18.00 \\
\hline \hline
\end{tabular}

\section{The reduction and analysis}

On the basis of the measurements of 18 comparison stars we have constructed the sensitometric characteristic for each plate, with the help of which the photographic magnitudes of the individual stars studied were determined. The results obtained that way for 17 investigated stars, along with the Asiago magnitudes are summarized chronologically in Table 2. The standard deviations of sensitometric characteristics of each plate are in the interval $<0.03-0.27>m_{\mathrm{pg}}$. with mean value $0.13 m_{\mathrm{pg}}$. The cross-reference between star No., MS designation and catalogue one (provided it has already been adopted for a given star) according to the GCVS and IBVS (Kholopov et al. 1987) is given in Table 3 as well as the equatorial coordinates for the epoch J1950.0 for each star, which is compatible with the GCVS. In this table are shown the basic characteristics of the observational material for each star: the number of data points and interval of magnitudes obtained at the Asiago Observatory (A) and Skalnaté Pleso Observatory (SP) respectively. We have performed the period analysis of both the data groups A and SP respectively. Some data were omitted for the period analysis on the basis of the quality of the photographic plates. We have used the method of Fourier analysis (Deeming 1975; Kurtz 1985), the phase dispersion minimization method (Stellingwerf 1978) and the method of Fourier harmonic analysis (Andronov 1995) which fits the first harmonic term of a trigonometric polynome to the observed data. The advantage of the last method is the fact that it provides one not only with the determination of the parameters of variability alone, but also with the determination of the corresponding errors. A further result of the analysis is the determination of the moments of maxima (eventually minima) of the light changes through the routine by R. Komžík (1995), making use of the following methods: the parabola fit, the polynomial fit, the "center of mass", Kwee \& Van Woerden (1956), the sliding integrations and the tracing paper - polygonal line. The averages of values obtained through the individual methods were considered as the resulting values. The moments of maxima and minima are determined in the vicinity of the center of the observational interval. The type of the variability was determined as the last parameter, on the basis of the length of the period, the amplitude of light changes, the shape of LC and with the help of the characteristics defined in the GCVS.

\section{The results}

In this section, we summarize succinctly our results for all the stars under study. The LCs and phase diagrams of LCs are presented for each star. The power spectra (obtained by the Fourier method) or the periodograms (obtained by the Stellingwerf method) are presented only for those stars, whose periods look ambiguous. In cases with unambiguous determination of periods, we have not considered as important to present the power spectra or periodograms. The same holds for cases of rather short periods of variability with very complex and unclear structure of the power specra. The assignation of type of variability is adopted from the GCVS. We interpret the light behaviour of selected objects.

\section{V375 Cygni}

The period of 180 days is indicated for this variable star in the GCVS. MSMM determined the period of 302 days for this object. The data taken in this paper represent $84(\mathrm{SP})$ values in the interval $<11.7-14.6>m_{\mathrm{pg}}$. The LC constructed from both groups of data is displayed in Fig. 1.

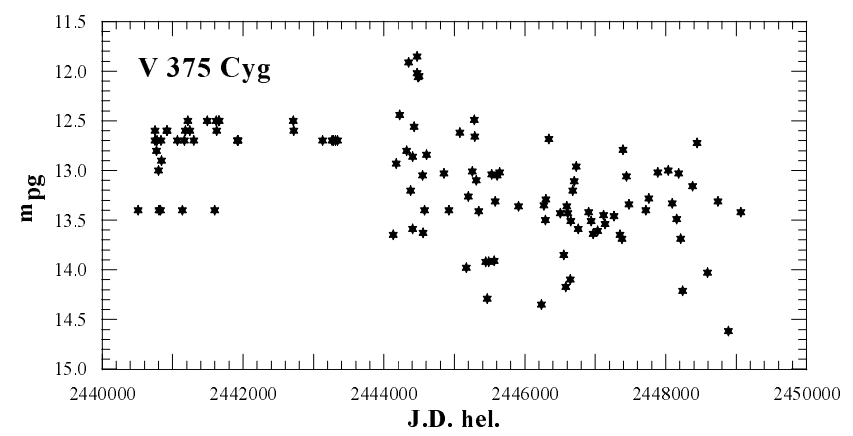

Fig. 1. The LC of V375 Cyg (file A + SP)

The period analysis of the combined data file $\mathrm{A}+\mathrm{SP}$ gave us no consistent results. In the next step we have divided the data in 3 groups: file A (JD 2440508 - 2443337), file SP, 1st part (JD $2444130-2445912$ ) and file SP, 2nd part (JD 2446236-2449119). The period analysis by Fourier method gave us following periods 298, around 280 and unambiguous 336 days for $\mathrm{A}, \mathrm{SP} / 1$ and $\mathrm{SP} / 2$ data respectively. This result suggests the semiregular character of the light changes. We classify V375 Cygni as a late-type semiregular variable. The most actual data file $(\mathrm{SP} / 2)$ is depicted in the phase diagram with period 335.9 days in Fig. 2. We have determined the epoch of maximum as $\mathrm{JD}_{\max }=2447395.0 \pm 3.7$ days. 


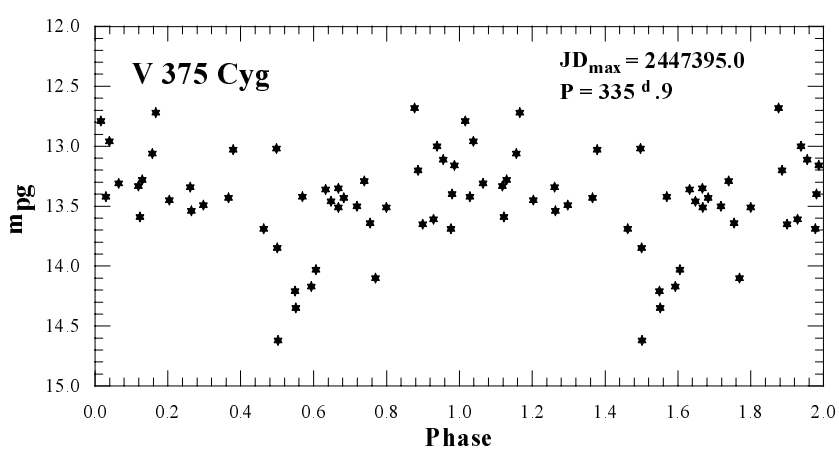

Fig. 2. The phase diagram of the LC of V375 Cyg (file SP, 2nd part)

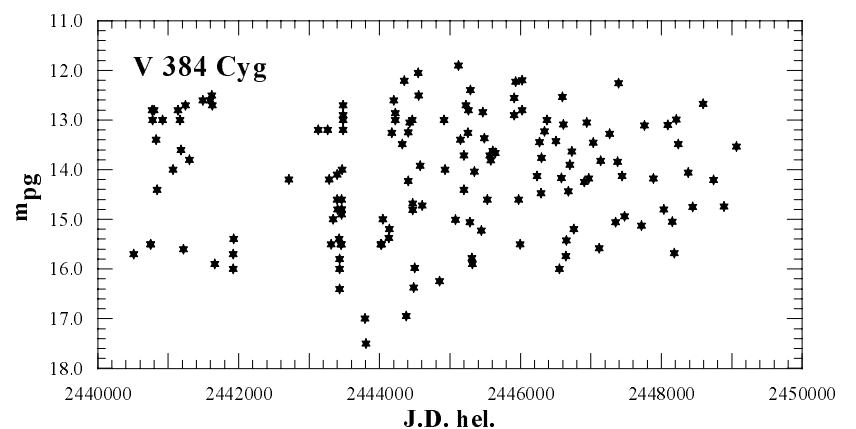

Fig. 3. The LC of V384 Cyg (file A + SP)

\section{V384 Cygni}

The period of 120 days is indicated for this star in the GCVS. MS determined the period of 117 days for the star. Our data represent 84 (SP) values in the interval $<12.1-17.0>m_{\mathrm{pg}}$. The analysis of the combining data $(\mathrm{A}+\mathrm{SP})$ consisting of 157 observed magnitudes in the brightness variations interval $<12.1-17.5>m_{\mathrm{pg}}$ (the LC is depicted in Fig. 3) has revealed an interesting result. Two statistically significant periods whose values are very close to each other appear in the periodogram: $P_{1}=118.1 \pm 0.1$ and $P_{2}=114.5 \pm 0.1$ and their first harmonics. This could suggest that there are two real periods existing simultaneously or that a subsequent period change occurred there. This possibility has been verified by the subdivision of the data into 4 subfiles which have been further analyzed individually (at the price of the lower accuracy of the results obtained). The results of the subsequent analysis of the individual parts of the LC are as follows:

File A, 1st part (JD 2440508 - 2443483) $P=117.2 \pm 0$ d 1 File A, 2nd part (JD 2443789-2446378) $P=114.8 \pm 0.1$ File SP, 1st part (JD 2444130-2446553) $P=114.9 \pm 0$ d 1 File SP, 2nd part (JD 2446584-2449119) $P=117^{\mathrm{d}} 3 \pm 0^{\mathrm{d}} \cdot 1$. The corresponding power spectra are shown in Fig. 4 and phase diagrams are displayed in Figs. 5-8. The errors of periods are round and correspond to the highest limits.

These results clearly suggest that the period of the light changes in V384 Cyg was changing during the time interval investigated: first it was shortening from 117 days

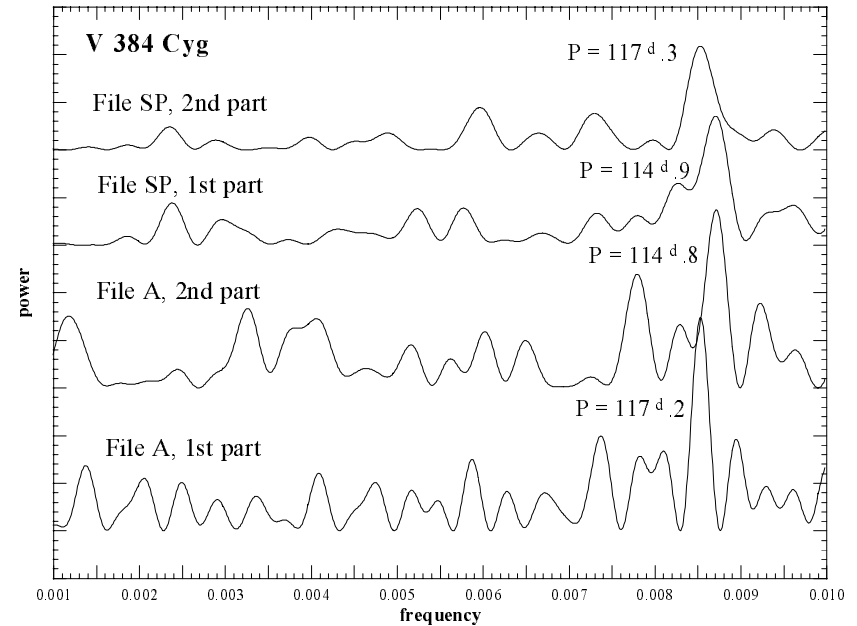

Fig. 4. Power spectra of V384 Cyg of individual data files

to 115 days in the middle of the observational interval and then it was increasing in length back to the original value of 117 days. The time interval of the period change is approximately 16 years. This is in a good accordance with the value calculated on the basis of the difference of the inverse values of periods 117.2 and 114.9 . We have determined the variability of this star to be Mira-type.

This result also demonstrates the fact that, through the use of the appropriate means of the data reduction/processing and analysis, it is possible to obtain such detailed information even from the photographic photometry which otherwise suffers from the burden of substantial systematic errors.

\section{V523 Cygni}

The period of light changes of 373.4 days is indicated for this star in the GCVS. MSMM present the period of 376.3 days. Combining the data files A and SP (LC is depicted in Fig. 9), we have obtained 101 values in the interval $<11.6-18.0>m_{\mathrm{pg}}$, the analysis of which provided us with the unambiguous period of $P=379.4 \pm 0 \mathrm{~d} 8$. On the basis of the behaviour of the LC, whose phase diagram is displayed in Fig. 10, we have determined the type of variability Mira and $\mathrm{JD}_{\max }=2444458.4 \pm 3.3$ days.

\section{MS 8}

There is no definitive designation for this star as yet, neither in The 68th Name-List of Variable Stars (Kholopov et al. 1987). MS did not succeed in the determination of any characteristic of the variability of this star. This has to do also with the small amplitude of the light changes, as well as with the irregular coverage of the interval investigated (see LC in Fig. 11). The SP data represent 84 values from the interval $<12.1-13.4>m_{\mathrm{pg}}$. The period analysis of this file has very probably excluded any long period. The period of $P=0 \mathrm{~d} 2534 \pm 0 \mathrm{~d} 0003$ has revealed itself as the most probable one. We must note that the power spectrum has a very small amplitude and is too 
Table 3. The basic characteristics of investigated stars and observational material

\begin{tabular}{ccccccccc}
\hline \hline Star No. & MS & GCVS/IBVS & $\alpha_{1950.0}$ & $\delta_{1950.0}$ & $n(\mathrm{~A})$ & $\Delta m_{\mathrm{pg}}(A)$ & $n(\mathrm{SP})$ & $\Delta m_{\mathrm{pg}}(S P)$ \\
\hline 1 & & V 375 Cyg & 204532 & +3542.1 & 35 & $12.5-13.4$ & 84 & $11.9-14.6$ \\
2 & & V 384 Cyg & 204439 & +3448.6 & 73 & $12.2-17.5$ & 84 & $12.1-17.0$ \\
3 & & V 523 Cyg & 210004 & +3506.9 & 71 & $11.8-18.0$ & 30 & $11.6-17.9$ \\
4 & 8 & - & 204103 & +3519.3 & 54 & $12.8-13.7$ & 84 & $12.1-13.4$ \\
5 & 18 & V 1838 Cyg & 204345 & +3633.2 & 52 & $12.0-18.0$ & 44 & $14.0-17.5$ \\
6 & 38 & V 1854 Cyg & 204617 & +3641.5 & 53 & $14.2-15.2$ & 84 & $13.4-15.5$ \\
7 & 40 & V 1856 Cyg & 204621 & +3516.1 & 82 & $14.2-15.7$ & 87 & $13.0-15.0$ \\
8 & 51 & V 1863 Cyg & 204742 & +3702.6 & - & - & 82 & $13.3-15.5$ \\
9 & 53 & V 1864 Cyg & 204809 & +3718.9 & 55 & $14.3-18.0$ & 35 & $14.5-16.9$ \\
10 & 63 & V 1868 Cyg & 204917 & +3642.5 & 55 & $14.3-18.5$ & 20 & $13.5-16.6$ \\
11 & 66 & V 1871 Cyg & 204942 & +3505.9 & 57 & $13.6-15.6$ & 88 & $12.5-15.5$ \\
12 & 73 & V 1877 Cyg & 205042 & +3412.3 & 56 & $13.8-15.2$ & 66 & $13.1-14.8$ \\
13 & 83 & V 1886 Cyg & 205416 & +3610.3 & 51 & $14.1-15.6$ & 85 & $13.4-15.2$ \\
14 & 86 & V 1889 Cyg & 205509 & +3356.5 & 53 & $14.3-15.8$ & 49 & $13.4-15.6$ \\
15 & 91 & - & 205729 & +3626.6 & 52 & $13.0-15.3$ & 81 & $12.4-15.4$ \\
16 & 95 & V 1894 Cyg & 205843 & +3353.3 & 42 & $12.1-13.3$ & 18 & $12.3-13.4$ \\
17 & 99 & - & 210303 & +3528.1 & 22 & $14.0-16.4$ & 12 & $13.9-16.7$ \\
\hline \hline
\end{tabular}

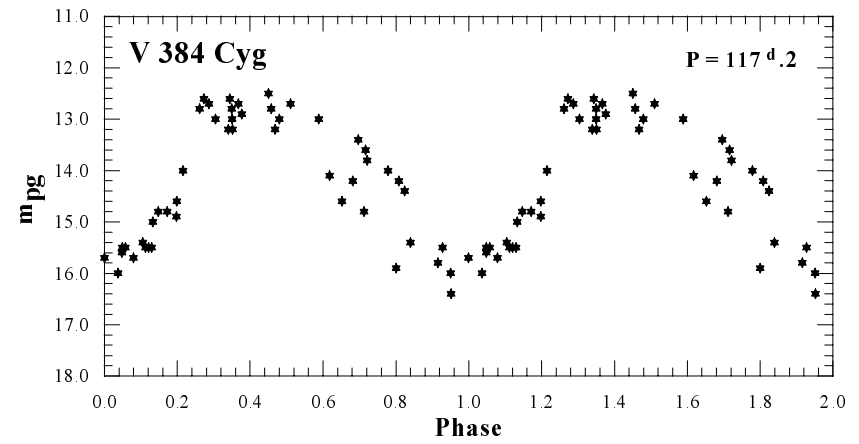

Fig. 5. The phase diagram of the LC of V384 Cyg (file A, 1st part)

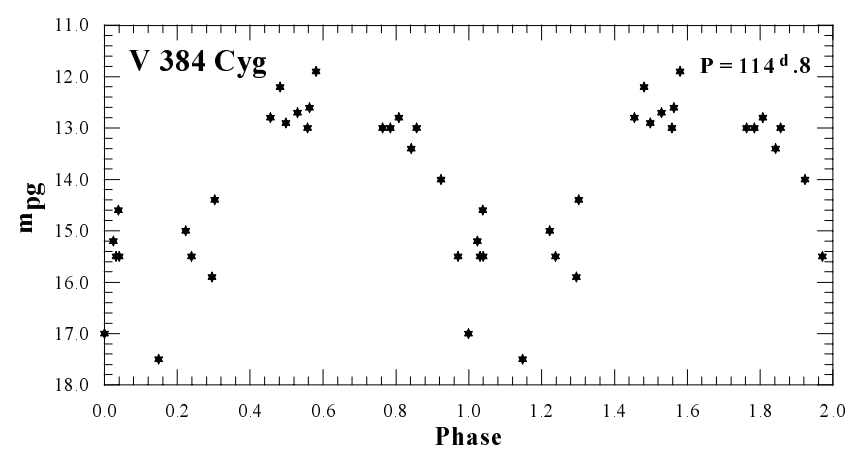

Fig. 6. The phase diagram of the LC of V384 Cyg (file A, 2nd part)

noisy to get definitive results. The corresponding epoch of maximum is $\mathrm{JD}_{\max }=2446599.129 \pm 0.009$ days. On the basis of the behaviour of the LC, whose phase diagram is displayed in Fig. 12, we have determined the variability of this star to be of the RR Lyrae-type. More accurate data are inevitable for the verification of the above period.

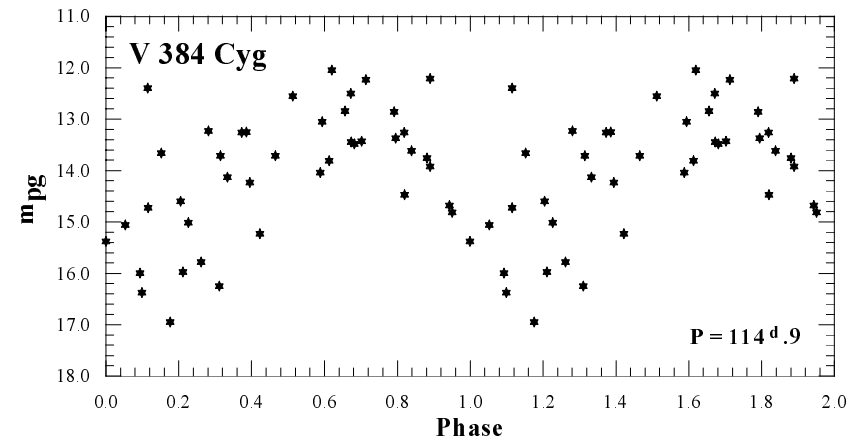

Fig. 7. The phase diagram of the LC of V384 Cyg (file SP, 1st part)

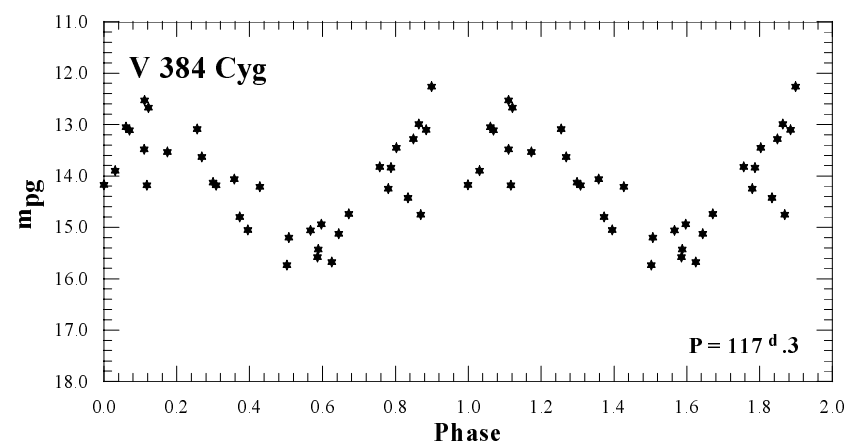

Fig. 8. The phase diagram of the LC of V384 Cyg (file SP, 2nd part)

\section{V1838 Cygni}

MS determined the period of this variable star to be of 343 days. Adding $44 \mathrm{SP}$ values in the interval $<14.0-17.5>m_{\mathrm{pg}}$ to the A data (the LC is depicted in Fig. 13), we were able to improve the accuracy of the original period obtaining the value of $P=340.7 \pm 0$ d 8 and to determine the epoch of maximum 


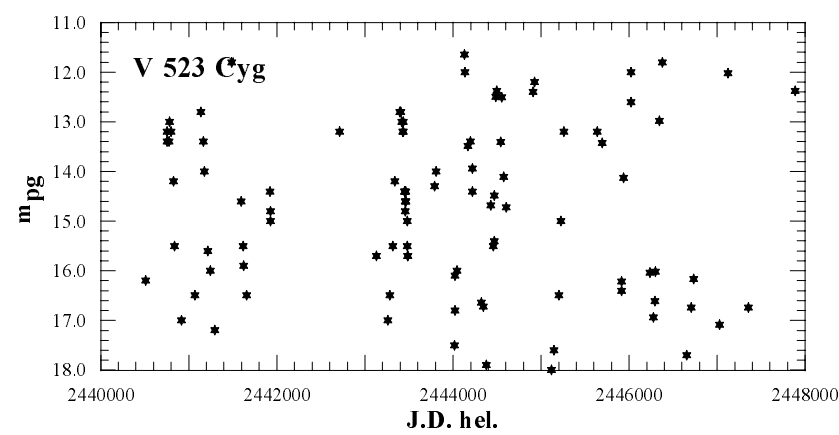

Fig. 9. The LC of V523 Cyg (file A + SP)

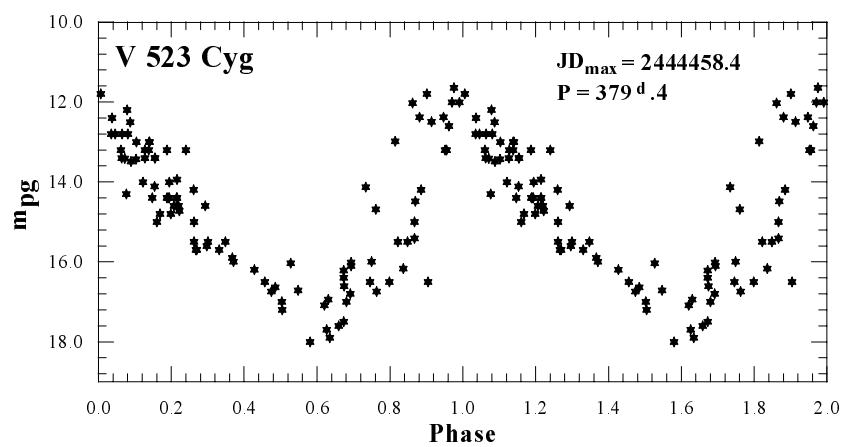

Fig. 10. The phase diagram of the LC of V523 Cyg (file A + $\mathrm{SP})$

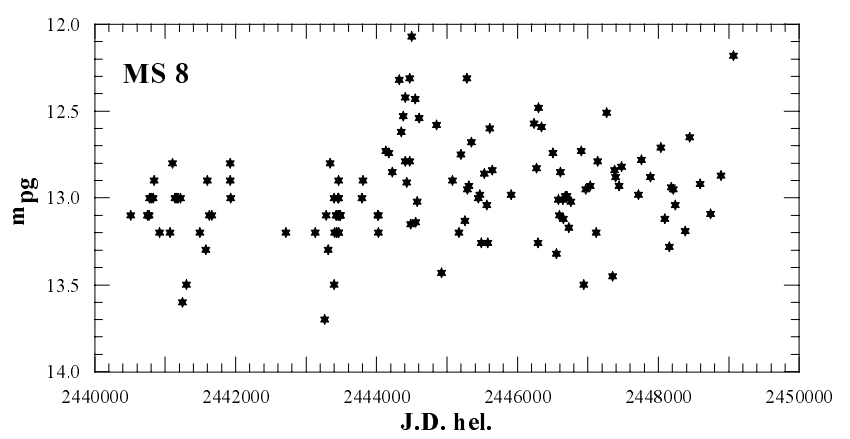

Fig. 11. The LC of MS 8 (file A + SP)

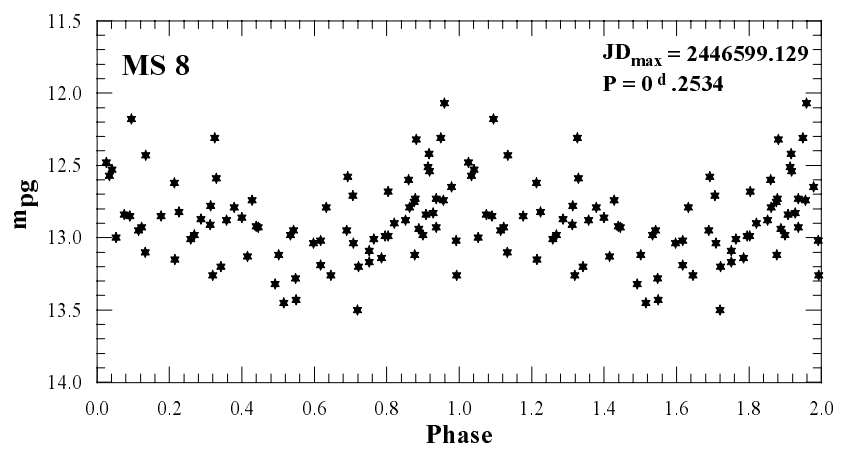

Fig. 12. The phase diagram of the LC of MS 8 (file SP)
$\mathrm{JD}_{\max }=2443967.7 \pm 5.7$ days. The corresponding phase diagram (Fig. 14) clearly implies the variability of this star to be of Mira-type. The brightest value in the $\mathrm{LC}$ is from file $\mathrm{A}$.

\section{V1854 Cygni}

Only the type of variability SR is indicated for this object by MS. The LC has been constructed on the basis of 135 values $m_{\mathrm{pg}}$ in the interval $<13.4-15.5>m_{\mathrm{pg}}$. This LC is displayed in Fig. 15. In spite of a substantial number of the observed points, the result of the period analysis is very complicated. The periodogram (Fig. 16) exhibits a complex structure consisting of many peaks, all of them corresponding to possible periods. The only real period is $P=106.5$, the remaining peaks are the harmonics of the basic period or the amplified aliases corresponding to the superposition of the period 106.5 and one-year period in the data distribution. The amplification of the aliases is caused by the fact that the brightness of the star is changing in a rather narrow interval of values and the signal-to-noise ratio decreases. We have determined the epoch of maximum as $\mathrm{JD}_{\max }=2446240 \pm 5$ days, and the type of variability of this star to be that of a semiregular variable. The very noisy phase diagram of the LC (probably connected with semiregular character of variability) is displayed in Fig. 17.

\section{V1856 Cygni}

The following parameters are given for this star by MS: $P=1$ d $99918, \mathrm{JD}_{\min }=2445640.412$ days and the type of variability EA (Algol type eclipsing binary). Through the combination of the data files from Skalnaté Pleso and Asiago, we have obtained 169 data points in the interval of the brightness changes $<13.0-15.7>m_{\mathrm{pg}}$ covering the time span of 24 years (LC is depicted in Fig. 18). Such an extension of the observational material creates good environment for the improvement of the accuracy of the object's parameters.

The period analysis has provided us with the more precise result: $P=1.999201 \pm 0.000062$ for $\mathrm{JD}_{\min }=2448785.201 \pm 0.069$ days. The phase diagram of the relevant LC (Fig. 19) confirms the eclipsing character of the light variations of this variable star. The primary minima are well visible. It is possible to find a slight sign of the secondary minima at about the phase 0.6 (the large scatter of the data makes the deeper analysis impossible). The mild wave-like shape of the quasi-constant phase of the light curve between the primary minima could be caused by the ellipticity of the components or by the presence of circumstellar matter.

\section{V1863 Cygni}

MS have only determined the type of variability LB (longperiod variable) for this star. In further investigation of this object, we have relied only on the SP data file consisting of 82 values in the interval $<13.3-15.5>m_{\mathrm{pg}}$. 


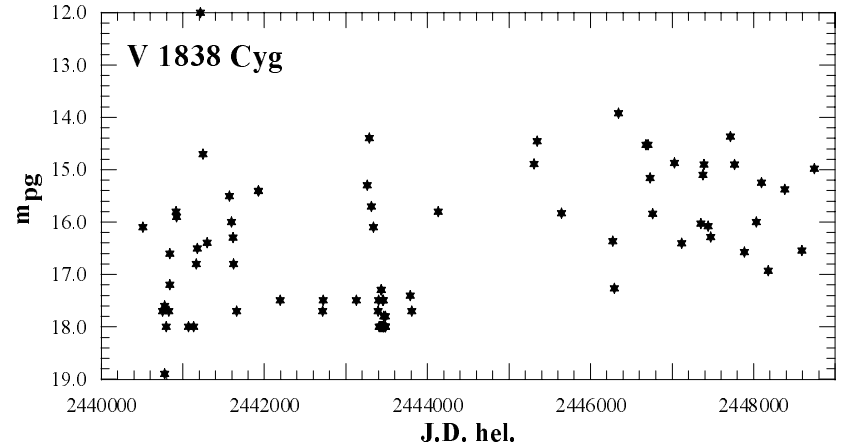

Fig. 13. The LC of V1838 Cyg (file A + SP)

The relevant A file was not available to us. The LC constructed from SP data is displayed in Fig. 20. We have searched as wide interval of presumable period values as possible $(0.1-1000$ days $)$. The period of $P=7.322 \pm 0.002$ has revealed itself to be the most probable one. The corresponding epoch of maximum is $\mathrm{JD}_{\max }=2446499.7 \pm 0.3$ days. On the basis of this result, as well as judging from the shape of the LC (the corresponding phase diagram is displayed in Fig. 21), we can exclude the originally determined type of variability. Instead of it, we suggest the Cepheid-type classification for this object as the more plausible one. The poor quality of the observational data produces the power spectrum with small signal to noise ratio. More observational data of higher accuracy (e.g. CCD photometry) are inevitable in order to determine the parameters of variability of this star definitively.

\section{V1864 Cygni}

MS, have determined its period to be of 448 days, suggesting that the object belongs to the Mira-type variable. Through the substantial extension of the observational interval ( $\mathrm{LC}$ constructed on the basis of 90 values $m_{\mathrm{pg}}$ in the brightness interval $<14.3-18.0>$ is shown in Fig. 22), we succeeded in the improvement of the precision of these values. The new variability parameters are as follows: $P=463.2 \pm 0.4$ and $\mathrm{JD}_{\max }=2443494.3 \pm$ 7.0 days. The length of the period and the shape of the phase diagram (Fig. 23) confirm the classification of the type of variability as Mira. The larger scatter of the data around the minimum of the LC is caused by the lower limiting magnitude of the plates from Skalnaté Pleso Observatory which did not allow to record the minimum brightnesses of this star. The waving on the ascending branch of the LC could be attributed to the transition of a shock wave through the extended atmosphere of the star (Kudashkina \& Rudnitskij 1994).

\section{V1868 Cygni}

MS determined the period of $406 \mathrm{~d} 5$ for this star, the object belonging to the Mira-type of variables. The SP file contains 20 values in the interval $<13.5-16.6>m_{\mathrm{pg}}$.

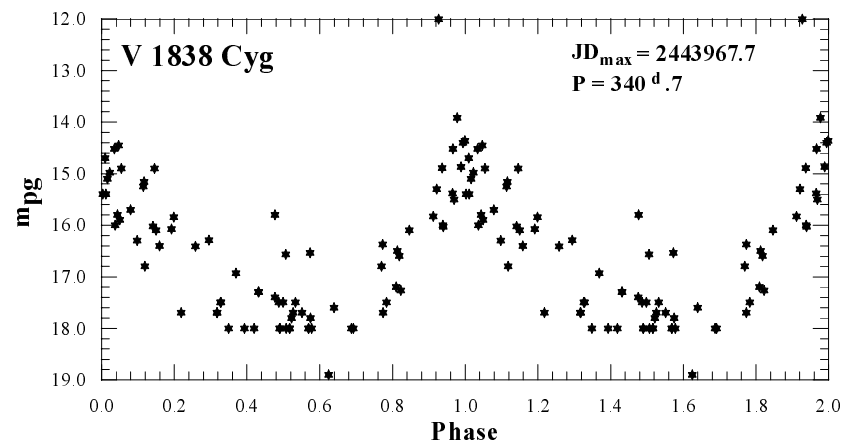

Fig. 14. The phase diagram of the LC of V1838 Cyg (file A + $\mathrm{SP})$

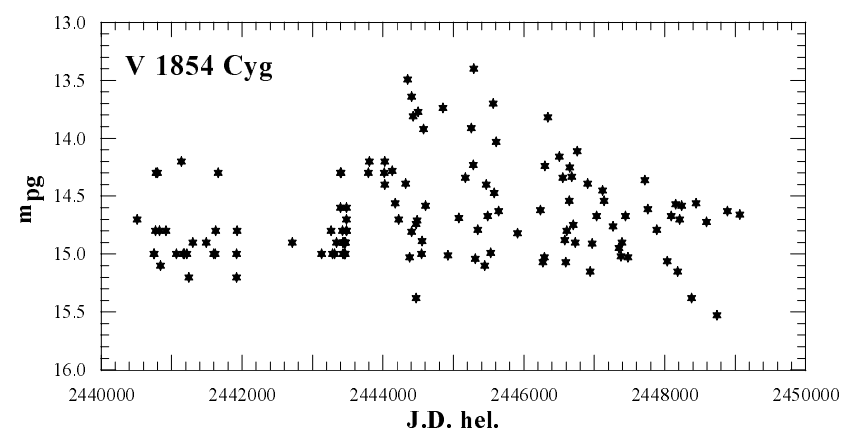

Fig. 15. The LC of V1854 Cyg (file A + SP)

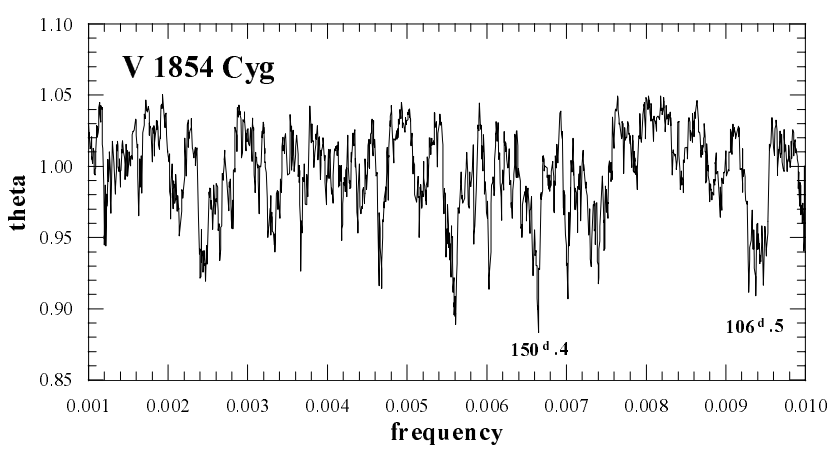

Fig. 16. The periodogram of V1854 Cyg (file SP)

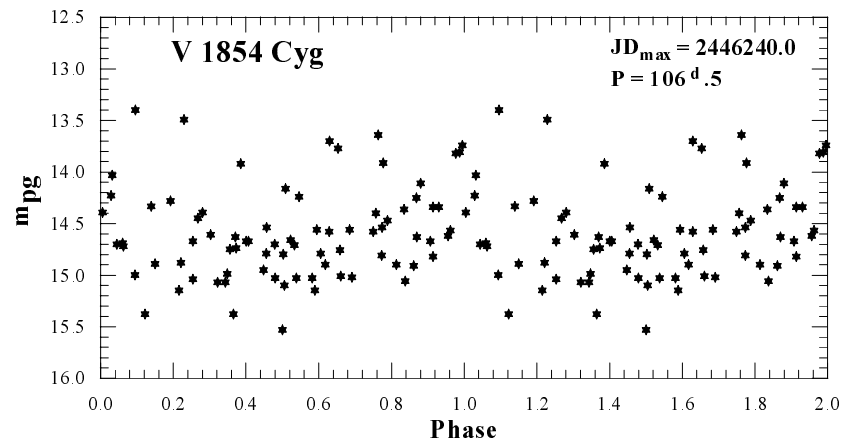

Fig. 17. The phase diagram of the LC of V1854 Cyg (file SP) 


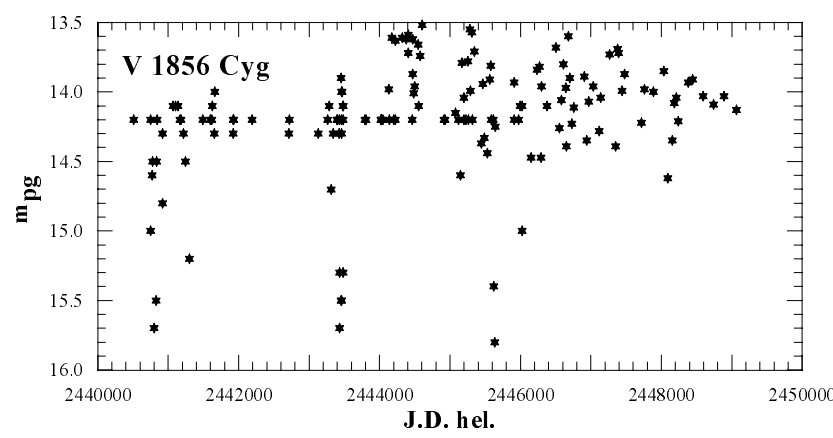

Fig. 18. The LC of V1856 Cyg (file A + SP)

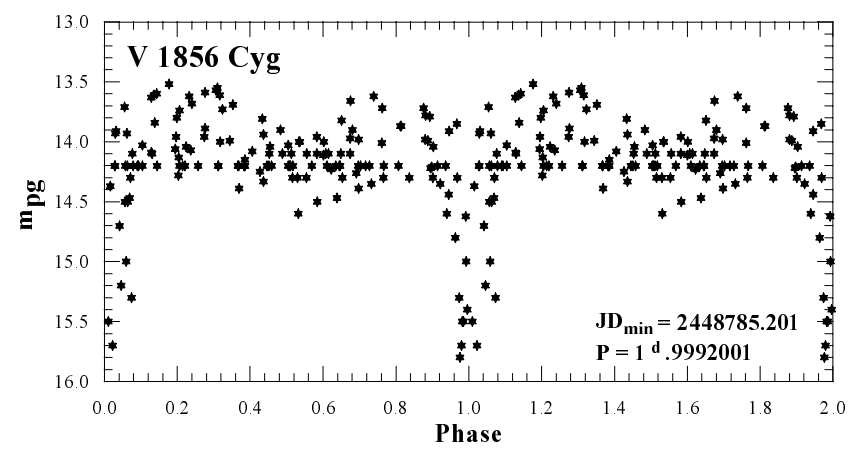

Fig. 19. The phase diagram of the LC of V1856 Cyg (file A + $\mathrm{SP})$

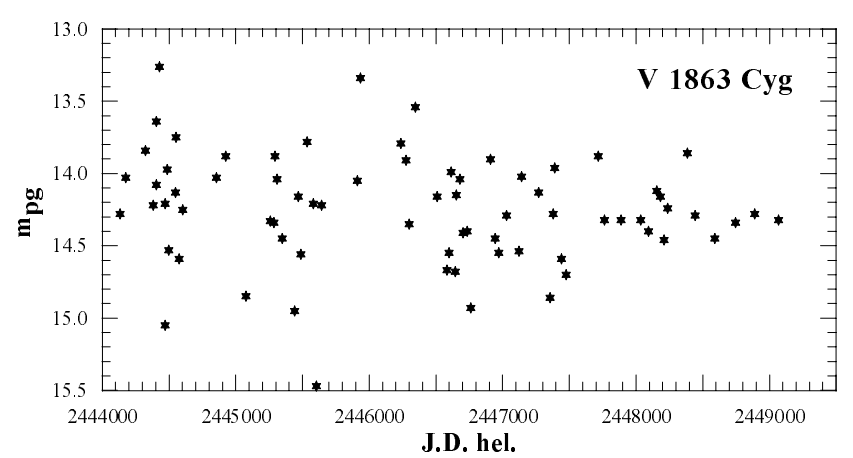

Fig. 20. The LC of V1863 Cyg (file SP)

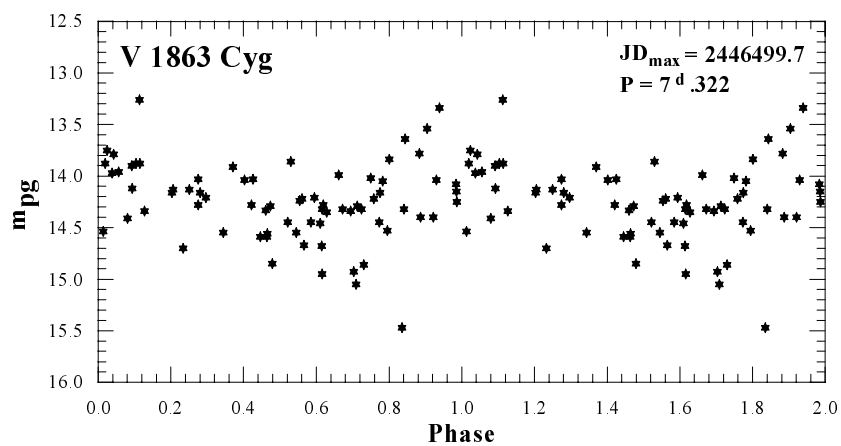

Fig. 21. The phase diagram of the LC of V1863 Cyg (file SP)

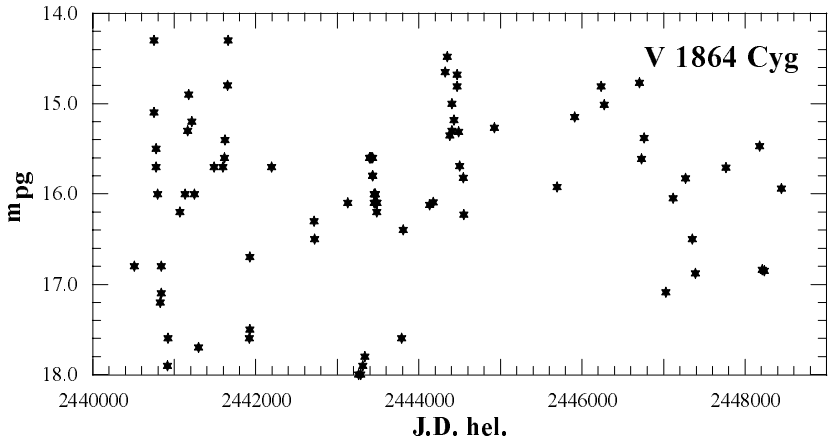

Fig. 22. The LC of V1864 Cyg (file A + SP)

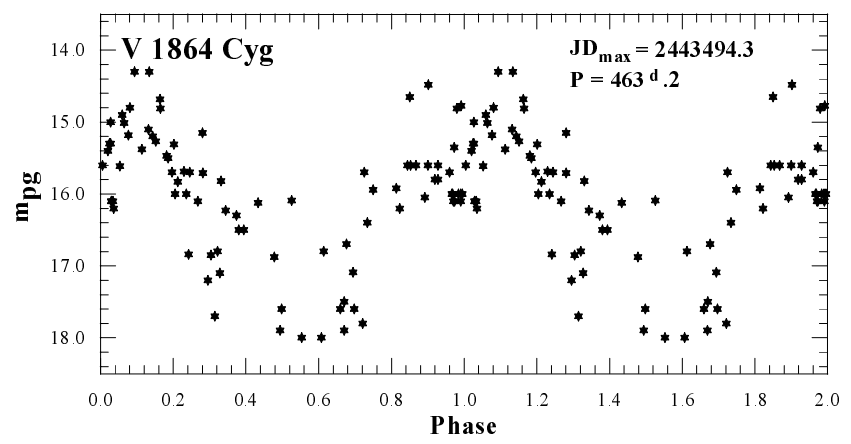

Fig. 23. The phase diagram of the LC of V1864 Cyg (file A + $\mathrm{SP})$

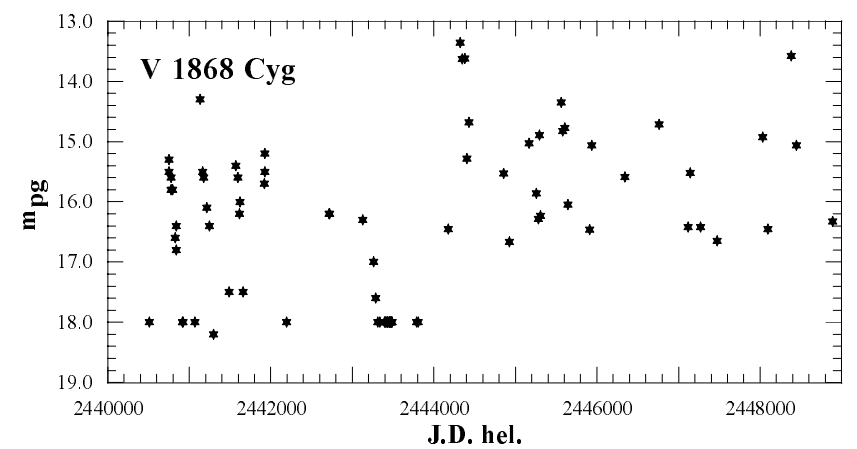

Fig. 24. The LC of V1868 Cyg (file A + SP)

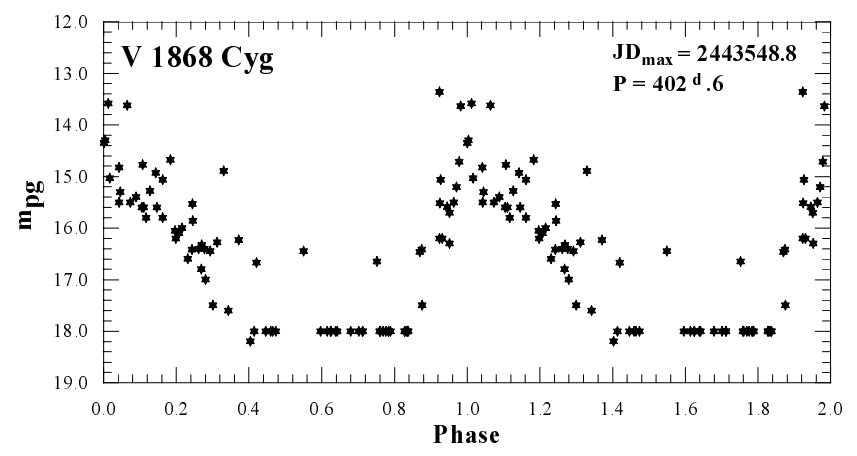

Fig. 25. The phase diagram of the LC of V1868 Cyg (file A + $\mathrm{SP})$ 


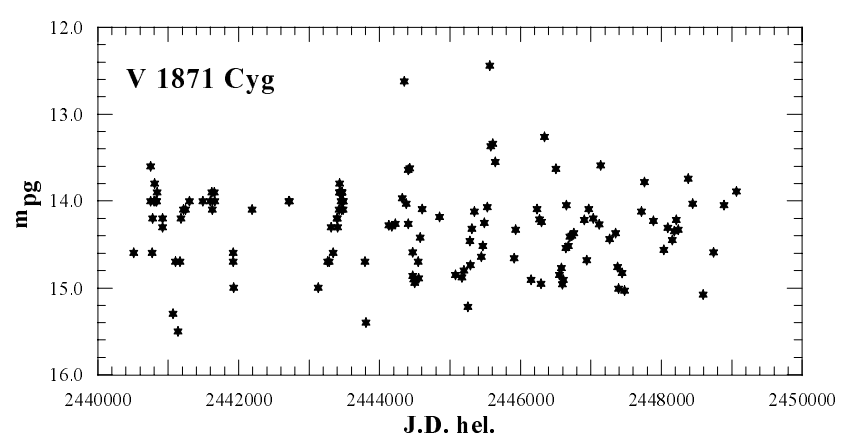

Fig. 26. The LC of V1871 Cyg (file A + SP)

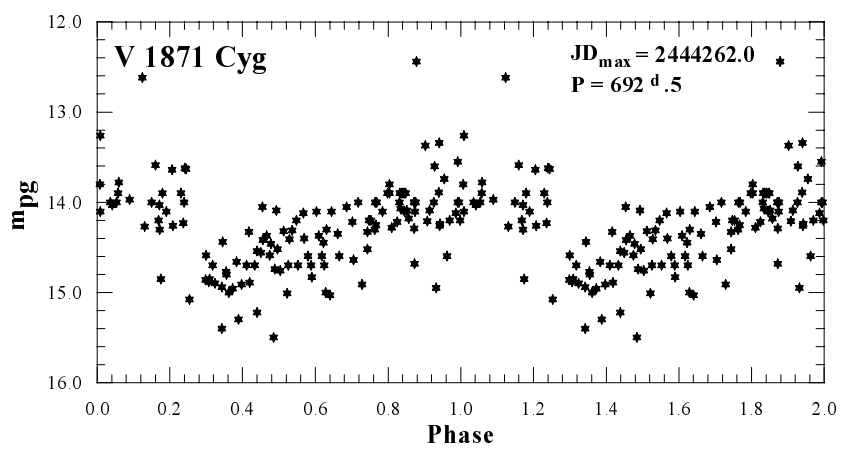

Fig. 27. The phase diagram of the LC of V1871 Cyg (file A + $\mathrm{SP})$

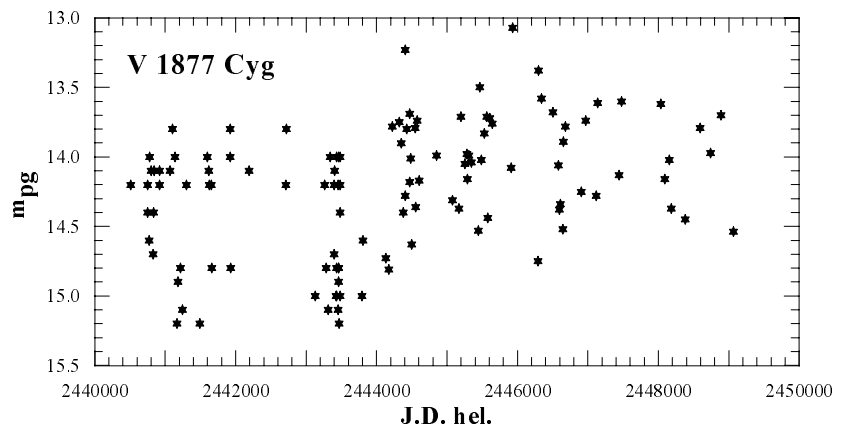

Fig. 28. The LC of V1877 Cyg (file A + SP)

Through the period analysis of the combined data A + SP (LC is shown in Fig. 24), we have obtained unambiguous results based on the power spectrum with only one significant period $P=402$ d $6 \pm 0$ d 8 . We have determined the epoch of maximum $\mathrm{JD}_{\max }=2443548.8 \pm 4.6$ days. This period represents slight improvement of the originally determined one. The corresponding phase diagram displayed in Fig. 25 confirms the result obtained as well as the originally determined type of variability (Mira). The flat minimum in the phase diagram is not real - it is caused completely by the limiting magnitude of the photographic plates.

\section{V1871 Cygni}

MS and MSMM have determined the period of 674 days and a semi-regular type of variability of this star. As a result of the addition of our 88 values of magnitude in the interval $<12.5-15.5>m_{\mathrm{pg}}$, the time interval investigated has increased in length by a factor of 2.5 . The
LC (Fig. 26) of this star is constructed from both data files $(\mathrm{A}+\mathrm{SP})$. From the combined data, the period of the light changes was determined to be $P=692.5 \pm 4$ d 1 and the moment of maximum to be $\mathrm{JD}_{\max }=2444262.0 \pm 13.2$ days. The phase diagram (Fig. 27) exhibits waving on the ascending branch of the LC which is possibly explained by the transition of the shock wave through the extended envelope of the star (Kudashkina \& Rudnitskij 1994). The characteristics presented above correspond much better to the Mira-type with a smaller interval of light variations (M:), but on the basis of difference between the values of periods as well as scatter in the phase diagram, the semi-regular type of variability is not excluded.

\section{V1877 Cygni}

This star has caused substantial problems during the determination of the basic characteristics of its variability. MS and later MSMM did not determine the period, nor the type of variability. This star is a good demonstration of the fact that the extension of the observational interval through the addition of $66 \mathrm{SP}$ values in the interval $<13.1-14.8>m_{\mathrm{pg}}$ to the combined data file can provide one with a statistically sufficient file for the successful application of the methods of period analysis. Light behaviour of this star is shown in Fig. 28. In the region of long periods $(100-1000$ days $)$, no significant period was found; in the region of short periods, several periods were detected in the power spectra. The period $P=4.54091 \pm 0.00076$ reveals itself as the significant one. We have further determined the epoch of maximum $\mathrm{JD}_{\max }=2444183.904 \pm 0.156$ days. We conclude that the star exhibits Cepheid-type of variability. This classification is also confirmed by the phase diagram displayed in Fig. 29. Nevertheless, we note that the power spectrum in the interval of shorter periods has very complex structure. Therefore, we do not consider the resulting period to be the definitive one.

\section{V1886 Cygni}

In the MS and MSMM papers, the authors were unable to determine either the possible period, or the type of variability for this object. Our new $85 \mathrm{SP}$ values in the interval $<13.4-15.2>m_{\mathrm{pg}}$, which are more homogeneously distributed along the time interval investigated (see LC in Fig. 30), provided us with better observational material appropriate for the period analysis. Two periods in the vicinity of 260 days have resulted from the analysis. The corresponding periodogram is displayed in Fig. 31. We have selected the value $P=254.8 \pm 1$ d. 6 on the basis of the power spectrum as the basic one for a semi-regular variable star. This type of variability determined by us explains why the preceding authors did not succeed in the determination of any period. The epoch of maximum $\mathrm{JD}_{\max }=2446140.0 \pm 8.7$ days has been further determined by us. The phase diagram of the LC is displayed in Fig. 32. 


\section{V1889 Cygni}

MSMM determined the period of the variability of this star to be 612 days, classified it as a semi-regular variable and admitted as a second period 315 days. The SP file contains further 49 values in the interval $<13.4-15.6>m_{\mathrm{pg}}$. Combining this file with the A file (the LC is presented in Fig. 33), the interval under investigation has increased in length by a factor of 2.5. The period analysis of the combined data file did not confirm any long period. As for the higher number of possible shorter periods, we have selected the value $P=1.3311 \pm 0.0006$ as the most significant one. Nevertheless, the obstacle of unambiguous period determination is the very low signal to noise ratio in the power spectrum. The phase diagram constructed on the basis of the SP data is displayed in Fig. 34 . We have further determined the epoch of maximum $\mathrm{JD}_{\max }=2446092.26 \pm 0.35$ days. On the basis of the characteristics of the light changes obtained, we did not confirm the type of variability determined by MS. We suggest instead the Cepheid-type classification as the most appropriate one. It would be quite important to obtain the more complete light curve for this star using the CCD photometry, thereby verifying the results obtained.

\section{MS 91}

There are no parameters of variability for this object indicated in the literature. MSMM just note that the period should be shorter than 1 day. The LC (Fig. 35) has been constructed on the basis of 133 values $m_{\mathrm{pg}}$ in the interval of $<12.4-15.4\rangle$ and its shape confirms the shortperiod variations of this star. The following variability parameters were determined: $P=0 \mathrm{~d} 345372 \pm 0 \mathrm{~d} 000043$ for $\mathrm{JD}_{\max }=2447415.787 \pm 0.132$ days. The shape of the phase diagram of the data obtained at the Asiago Observatory looks interesting (Fig. 36). It exhibits two levels through which the star transits into the minimum of its brightness. Of special interest is that one which creates a wave-like feature or a hump on the descending branch of the curve. The origin of this hump could be explained by the differing brightnesses of the maxima in different cycles of the variations of this star (so-called Blazhko effect). The phase diagram of the combined data (Fig. 37) confirms such a type of change in shape of the LC by the more pronounced scatter of the data.

On the basis of these parameters, we have classified this object as the variable star of the RR Lyrae-type. Finally, we note that the star has also recently been studied by Andronov et al. (1994) and the parameters obtained by us are in very good agreement with their results.

\section{V1894 Cygni}

The variability of this star was originally determined by MS. In a more recent paper by MSMM, the authors have presented more precise characteristics of variability of this

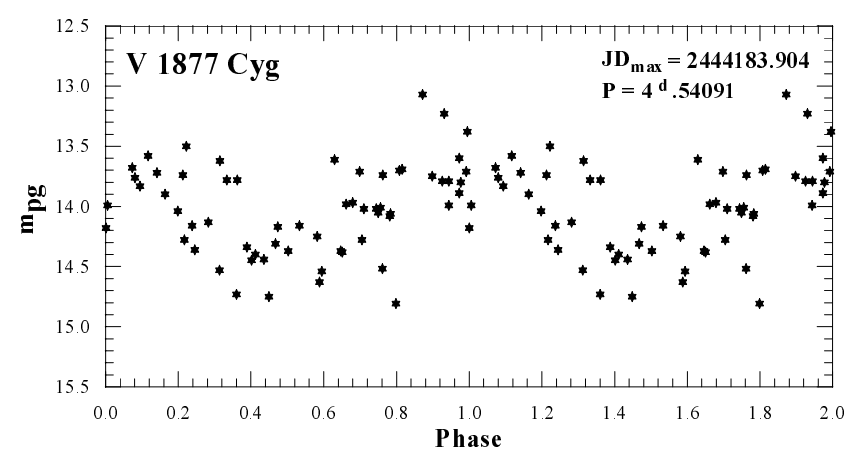

Fig. 29. The phase diagram of the LC of V1877 Cyg (file SP)

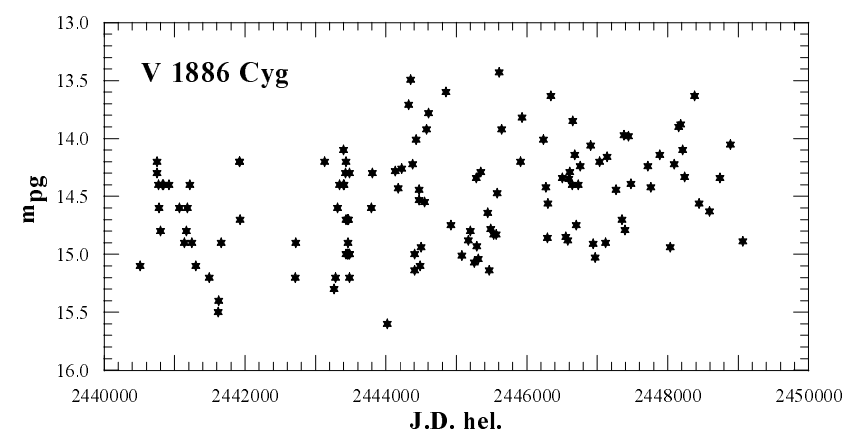

Fig. 30. The LC of V1886 Cyg (file A + SP)

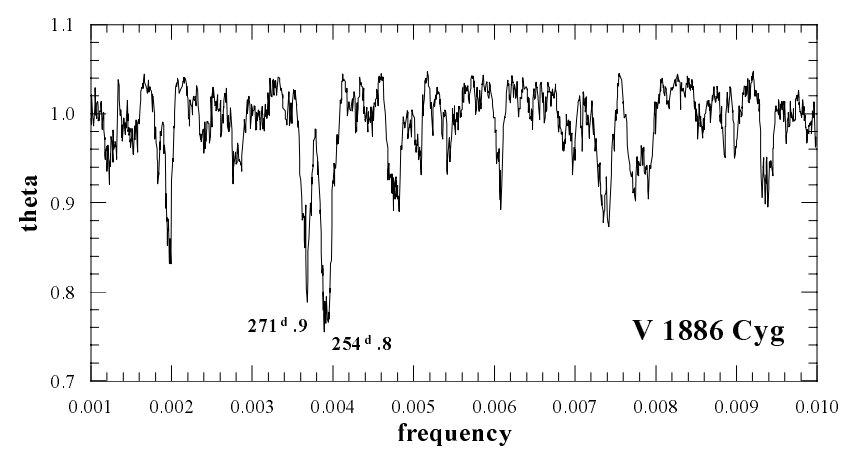

Fig. 31. The periodogram of V1886 Cyg (file SP)

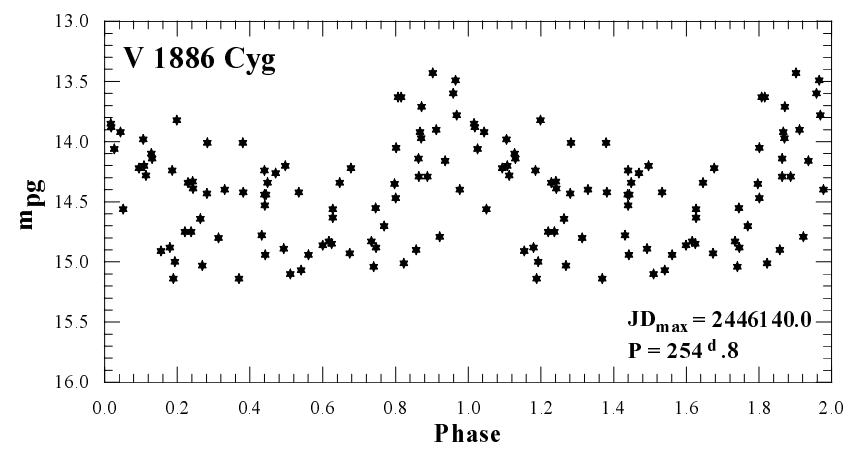

Fig. 32. The phase diagram of the LC of V1886 Cyg (file SP) 
Table 4. The resulting parameters for variable stars under investigation.

\begin{tabular}{ccllllll}
\hline \hline GCVS/IBVS/MS & $\Delta m_{\mathrm{pg}}$ & $P[\mathrm{~d}]$ & $\sigma(P)[\mathrm{d}]$ & $T_{\max }($ JDhel. $)$ & $\sigma\left(T_{\max }\right)[\mathrm{d}]$ & Type & Note \\
\hline V 375 Cyg & $11.9-14.6$ & 335.9 & 0.9 & 2447395.0 & 3.7 & SRA & 1 \\
V 384 Cyg & $12.1-17.5$ & $114.8-117.3$ & 0.1 & - & - & $\mathrm{M}$ & 2 \\
V 523 Cyg & $11.6-18.0$ & 379.4 & 0.8 & 2444458.4 & 3.3 & $\mathrm{M}$ & 3 \\
MS 8 & $12.1-13.7$ & 0.2534 & 0.0003 & 2446599.129 & 0.009 & RR Lyr & $4,5,6$ \\
V 1838 Cyg & $12.0-18.0$ & 340.7 & 0.8 & 2443967.7 & 5.7 & $\mathrm{M}$ & 3 \\
V 1854 Cyg & $13.4-15.5$ & 106.5 & 0.5 & 2446240.0 & 5.0 & SR & 4 \\
V 1856 Cyg & $13.0-15.7$ & 1.999201 & 0.000062 & 2448785.201 & 0.069 & EA & 3,7 \\
V 1863 Cyg & $13.3-15.5$ & 7.322 & 0.002 & 2446499.7 & 0.3 & CEP & $4,6,8$ \\
V 1864 Cyg & $14.3-18.0$ & 463.2 & 0.4 & 2443494.3 & 7.0 & M & 3 \\
V 1868 Cyg & $13.5-18.5$ & 402.6 & 0.8 & 2443548.8 & 4.6 & M & 3 \\
V 1871 Cyg & $12.5-15.6$ & 692.5 & 4.1 & 2444262.0 & 13.6 & M: & 3,8 \\
V 1877 Cyg & $13.1-15.2$ & 4.54091 & 0.00076 & 2444183.904 & 0.156 & CEP & $4,5,6$ \\
V 1886 Cyg & $13.4-15.6$ & 254.8 & 1.6 & 2446140.0 & 8.7 & SR & 4,5 \\
V 1889 Cyg & $13.4-15.8$ & 1.3311 & 0.0006 & 2446092.26 & 0.35 & CEP & $6,8,9$ \\
MS 91 & $12.4-15.4$ & 0.345372 & 0.000043 & 2447415.787 & 0.132 & RR Lyr & 4,5 \\
V 1894 Cyg & $12.1-13.4$ & 257.8 & 2.1 & 2443749.0 & 18 & M: & 8,9 \\
MS 99 & $13.9-16.7$ & - & - & - & - & SR & 10 \\
\hline \hline
\end{tabular}

Notes to Table 4:

1. the value of the period of variable star is close to the one originally published in MSMM,

2. the value of the period of variable star is changing irregularly in the interval indicated with the period of 16 years,

3. the improvement of the accuracy of the value of the period of variable star,

4. the determination of a new (as yet unknown) value of the period of variable star,

5 . the determination of a new (as yet unknown) type of variability,

6. more observational data are inevitable for the definitive determination of the period of variability,

7. the value of the epoch of minimum is presented,

8. the original classification was not confirmed, a new type of variability was determined,

9. the originally determined value of the period was not confirmed, a new value of the period was determined,

10. the unambiguous period of variable star was not determined.

For all the variable stars under investigation (except of V384 Cyg and MS 99, with respect to the character of their variability and the results found), new, more accurate values of the epoch of the maximum (eventually that of the minimum) were determined.

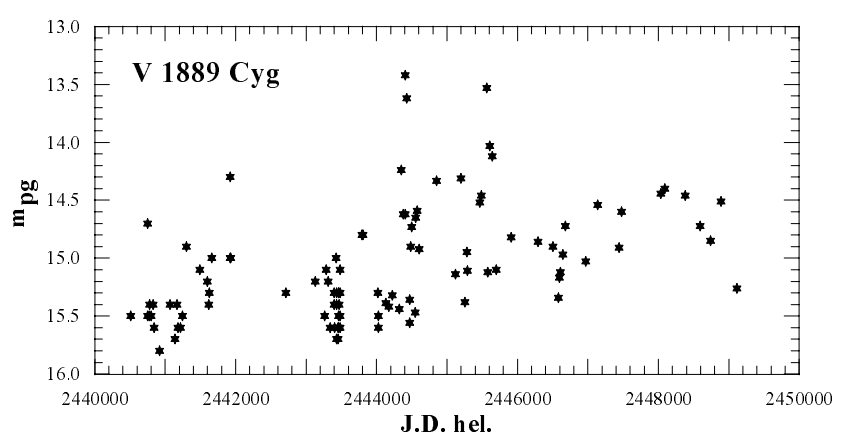

Fig. 33. The LC of V1889 Cyg (file A + SP)

star and determined its period to be $P=424$ days. The SP file extends the data available by further 18 values in the interval $<12.3-13.4>m_{\mathrm{pg}}$ and complete $\mathrm{LC}$ is displayed in Fig. 38. The period analysis of the combined $\mathrm{A}+\mathrm{SP}$ data has provided us with two significant periods in the vicinity of 479.4 and $255^{\mathrm{d}} .1$ (see periodogram shown

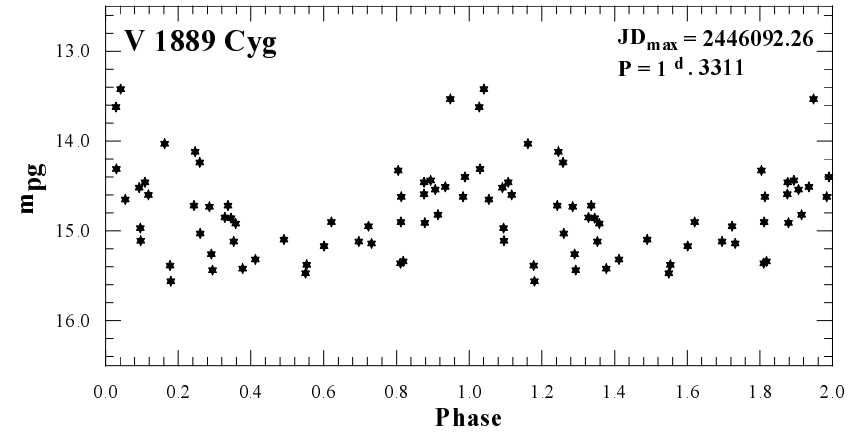

Fig. 34. The phase diagram of the LC of V1889 Cyg (file SP)

in Fig. 39), while we were not able, even with the help of the other methods, to decide which of these two periods is the real one. As the next step, we used an independent method of fitting the LC with a sinusoid. We have used both periods found for the trial sinusoids, finding that the 479.4 period does not fit the combined data file 


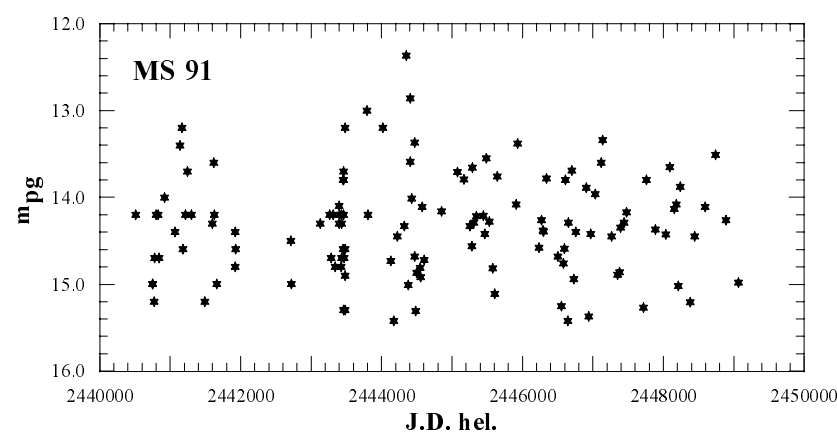

Fig. 35. The LC of MS 91 (file A + SP)

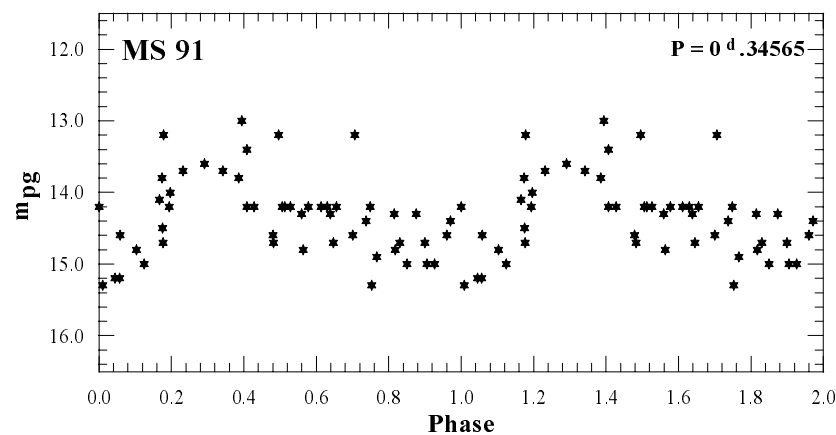

Fig. 36. The phase diagram of the LC of MS 91 (file A)

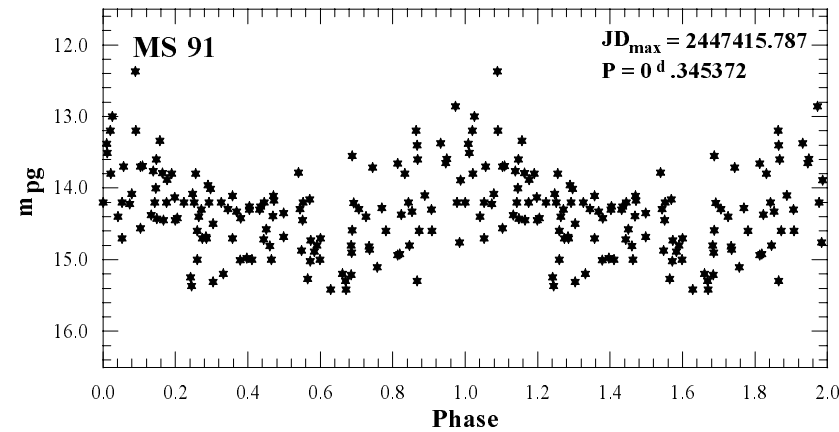

Fig. 37. The phase diagram of the LC of MS 91 (file A + SP)

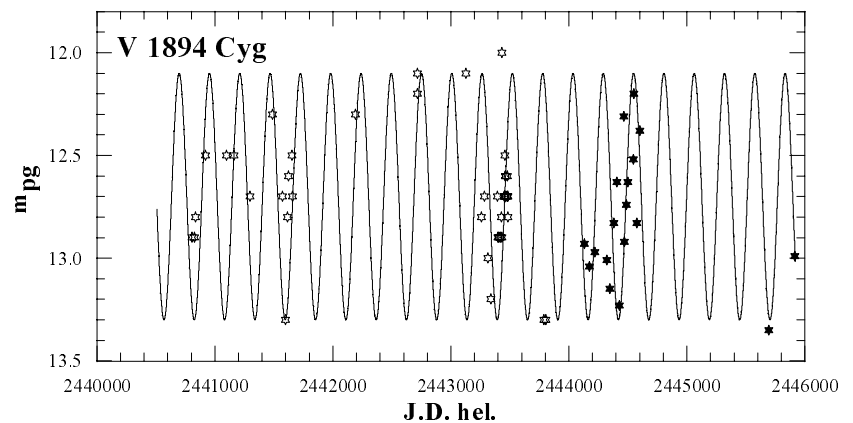

Fig. 38. The sinusoidal fit of the LC of variable star V1894 Cyg (file $\mathrm{A}+\mathrm{SP}$ ) with the period of 255.1

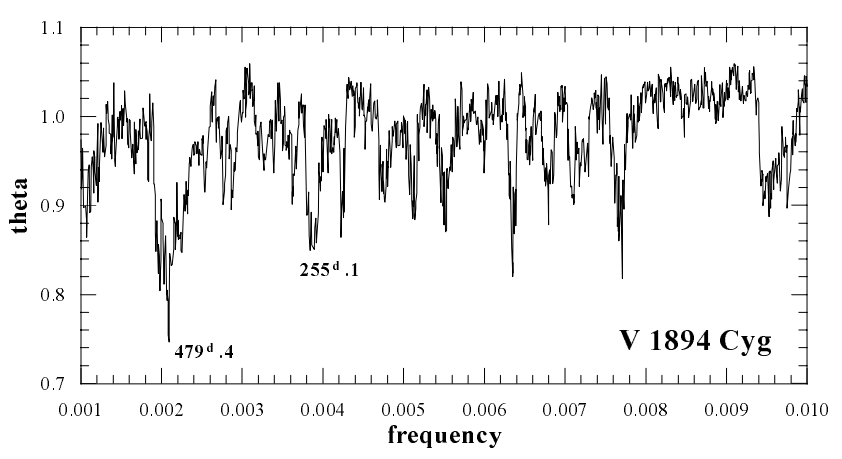

Fig. 39. The periodogram of V1894 Cyg (file A + SP)

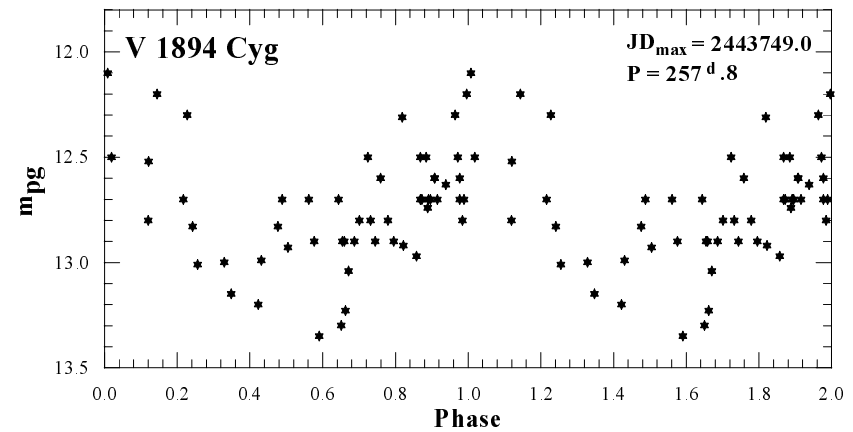

Fig. 40. The phase diagram of the LC of V1894 Cyg (file A + $\mathrm{SP})$

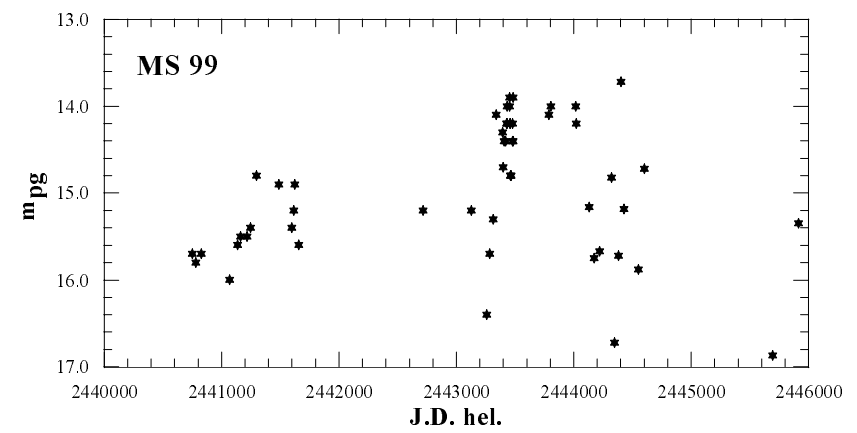

Fig. 41. The LC of MS 99 (file A + SP)

A + SP. Thus, we concluded that the real period is the shorter one. The process of fitting for this period is displayed in Fig. 38. After the weighing of the individual values of star's brightness in its LC, the period analysis has unambiguously determined the period $P=257^{\mathrm{d}} 8 \pm 2.1$ as the most significant one. The phase diagram with this period is displayed in Fig. 40. We have further determined the epoch of maximum $\mathrm{JD}_{\max }=2443749.0 \pm 18.0$ days and the Mira-type of variability.

\section{MS 99}

As for this variable star under study, MS obtained 22 values covering very inhomogeneously the time interval investigated. This has probably caused the fact that those authors did not find any other characteristics for this star except for its type of variability, specified by them as SR. 


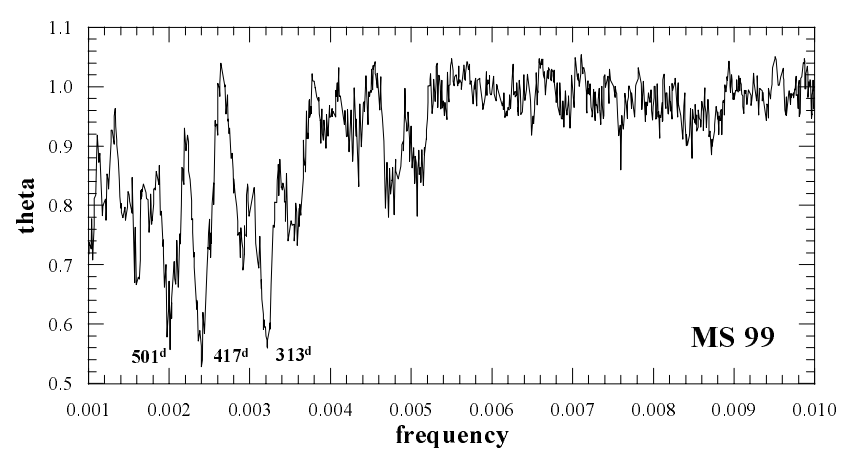

Fig. 42. The periodogram of MS 99 (file $\mathrm{A}+\mathrm{SP}$ )

The contribution of the SP data represents only 12 values in the interval $<13.9-16.7>m_{\mathrm{pg}}$. The LC of accumulated data is presented in Fig. 41. The period analysis of the combined A + SP data file has provided us with plenty of possible periods, of which the significant ones are located near the values of 313, 417 and 501 days (see Fig. 42). We did not detect any significant value in the region of short periods. In spite of the use of further independent methods, we were unable to decide which one of the periods we have found is the real one. The apparently irregular character of the variability of this star could represent a serious obstacle for any deeper study of its light behaviour.

\section{The summary of the results achieved}

All the results obtained through the analysis of the seventeen variable stars studied here are given in Table 4 . The parameters of variability of the investigated stars are presented there: GCVS/IBVS/MS designation, the interval of light changes, the period, the epoch of maximum (eventually the epoch of minimum) and the type of variability. As for the values of the period and the epoch, we present also the standard deviation of the determination of these quantities. These values represent the upper limits of the relevant errors. The note characterizes more closely the parameters obtained for the individual variable stars investigated by us or describes the relation of the results achieved by us to the results presented in the literature (GCVS, MS, MSMM). The short information about resulting parameters of the investigated variable stars without any discussion and interpretation has already been given to the astronomical community by Gális \& Hric (1998).

Acknowledgements. It is a pleasure to express our sincerest thanks to the Staff of the Asiago Astrophysical Observatory for the provision of the original observational material and for valuable advices during the measurement of the photographic plates. This work has been supported through the Slovak Academy of Sciences Grant No. 5016/98.

\section{References}

Andronov I.L., 1995 (private communication)

Andronov I.L., Chikrigin A.V., Kimeridze G.N., 1994, Odessa Astronomical Publications 7, part 1, 89

Deeming T.J., 1975, Astrophys. Space Sci. 36, 137

Gális R., Hric L., 1998, Inf. Bull. Variable Stars, No. 4565

Grygar J., Hric L., Chochol D., Mammano A., 1979, Bull. Astron. Inst. Czechosl. 30, 308

Hric L., Chochol D., Komžík R., 1993, Astrophys. Space Sci. 201, 107

Kholopov P.N., Samus' N.N., Frolov M.S., et al., 1985, Gen. Cata. Var. Stars, izdat. Nauka, Moskva

Kholopov P.N., Samus' N.N., Kazarovets E.V., Kireeva N.N., 1987, Inf. Bull. Var. Stars, No. 3058

Kohoutek L., 1969, Inf. Bull. Var. Stars, No. 384

Komžík R., 1995 (private communication)

Kudashkina L.S., Rudnitskij G.M., 1994, Odessa Astronomical Publications 7, part 1, 66

Kurtz O.W., 1985, MNRAS 213, 773

Kwee K.K., Van Woerden H., 1956, B.A.N 12, No. 464, 327

Margoni R., 1993 (private communication)

Margoni R., Stagni R., 1984, A\&AS 56, 87

Margoni R., Stagni R., Munari U., Marton S., 1989, A\&AS 81, 393

Stellingwerf R.F., 1978, ApJ 224, 953 